\title{
Implication of urine aquaporin-2 levels following cardiac surgery
}

\author{
Teruhiko Imamura ${ }^{1}$ [D
}

Received: 13 April 2020 / Accepted: 26 June 2020 / Published online: 29 June 2020

(c) Springer Japan KK, part of Springer Nature 2020

Keywords Heart failure · Aortic stenosis · Aortic valve replacement · Vasopressin

\section{To the editor}

In addition to the renin-angiotensin-aldosterone system and the sympathetic nerve system, the arginine vasopressin system is receiving great concern as a therapeutic target in patients with cardiovascular disease. Fujii and colleagues demonstrated that both plasma arginine vasopressin and urine aquaporin- 2 levels were elevated immediately after the surgical aortic valve replacement [1]. Both variables had a positive correlation with each other. They hypothesized that a urine aquaporin-2 level might be a potential biomarker of perioperative cardiac dysfunction. Several concerns should improve the implication of their findings.

Our team previously demonstrated that a reduced excretion of urine aquaporin-2 relative to plasma arginine vasopressin level might indicate impairment of kidney collecting duct [2]. Urine aquaporin-2 level may not be a good tool to assess cardiac function in patients with advanced chronic kidney disease, although such patients were not included in their study [1].

Second, our team could not demonstrate the implication of peri-procedural plasma arginine vasopressin level on clinical outcomes following trans-catheter aortic valve replacement, probably due to relatively maintained periprocedural hemodynamics in most patients [3]. Urine aquaporin-2 level might be useful particularly for the patients with relatively deteriorated hemodynamics including lowflow low-gradient aortic valve stenosis or decompensated heart failure.

\section{References}

1. Fujii M, Amitani R, Bessho R (2020) Perioperative urinary excretion of aquaporin-2 dependent upon vasopressin in cardiac surgery. Heart Vessels 35:712-728

2. Imamura T, Kinugawa K, Fujino T, Inaba T, Maki H, Hatano M, Yao A, Komuro I (2014) Increased urine aquaporin-2 relative to plasma arginine vasopressin is a novel marker of response to tolvaptan in patients with decompensated heart failure. Circ $\mathrm{J}$ 78:2240-2249

3. Kuwahara H, Imamura T, Sobajima M, Ueno H, Kinugawa $\mathrm{K}$ (2020) Regulation and clinical implication of arginine vasopressin in patients with severe aortic stenosis referred to trans-catheter aortic valve implantation. Medicina (Kaunas) 56:E165

Publisher's Note Springer Nature remains neutral with regard to jurisdictional claims in published maps and institutional affiliations.

This comment refers to the article available online at https://doi. org/10.1007/s00380-019-01533-8.

Teruhiko Imamura

teimamu@med.u-toyama.ac.jp

1 Second Department of Internal Medicine, University of Toyama, 2630 Sugitani, Toyama, Toyama 930-0194, Japan 